\title{
Correlations between eigenvalues of large random matrices with independent entries
}

\author{
J. D'Anna ${ }^{1}$ and A. Zee ${ }^{1,2}$ \\ ${ }^{1}$ Department of Physics, University of California, Santa Barbara, California 93106-9530 \\ ${ }^{2}$ Institute for Theoretical Physics, University of California, Santa Barbara, California \\ $93106-4030$
}

(August 16, 2018)

\begin{abstract}
We derive the connected correlation functions for eigenvalues of large Hermitian random matrices with independently distributed elements using both a diagrammatic and a renormalization group ( $R G$ ) inspired approach. With the diagrammatic method we obtain a general form for the one, two and three-point connected Green function for this class of ensembles when matrix elements are identically distributed, and then discuss the derivation of higher order functions by the same approach. Using the RG approach we re-derive the one and two-point Green functions and show they are unchanged by choosing certain ensembles with non-identically distributed elements. Throughout, we compare the Green functions we obtain to those from the class of ensembles with unitary invariant distributions and discuss universality in both ensemble classes.
\end{abstract}

PACS number(s): 05.40.+j 


\section{INTRODUCTION}

Of recent interest in random matrix theory has been the discovery and characterization of new universal behavior in the densities of, and correlations between the eigenvalues of various ensembles which may be relevant to the study of mesoscopic systems, disordered metals, and random surfaces [1].

In this paper we present an investigation for the existence of universal behavior in the correlation functions of a particular class of random matrix ensembles, those comprised of real symmetric or Hermitian matrices with individually distributed random elements. A notable example of an ensemble in this class is the first random matrix ensemble considered by Wigner [9], i.e. real symmetric matrices whose elements assume the values $\pm \sigma / \sqrt{N}$ with equal probability. Here we will refer to this class of theories as the "Wigner class".

It has been known for quite some time that in Wigner class ensembles the eigenvalue density is universal. More specifically, the averaged density of eigenvalues for an ensemble of matrices with individually identically distributed elements is the same for any chosen distribution, up to a scaling of the width of the spectrum. We define the averaged density of eigenvalues as

$$
\rho(\lambda)=\left\langle\frac{1}{N} \operatorname{Tr} \delta(\lambda-\varphi)\right\rangle
$$

where $\varphi$ is an $N \times N$ matrix and $\langle$.$\rangle denotes averaging over an ensemble of such matrices.$ In general, the ensemble average for an operator, $\mathcal{O}$, is obtained via the integral

$$
\langle\mathcal{O}(\varphi)\rangle \equiv \int d \varphi P(\varphi) \mathcal{O}(\varphi)
$$

where $P$ is a density function, to be defined in more detail later, which takes as its argument the matrix $\varphi$. For any Wigner Class ensemble one obtains the well known result

$$
\rho(\lambda)=\frac{2}{\pi a^{2}} \sqrt{a^{2}-\lambda^{2}}
$$

which is known as Wigner's "semi-circle" law. 
In this paper we will compare our results for "Wigner class" ensembles to those of another class, ensembles specified by a matrix density invariant under a symmetry group transformation. These "invariant class" (or "trace class" in the terminology of Refs. [1 -5]) ensembles have been shown to have non-universal eigenvalue densities. In particular, one finds the density of eigenvalues for an invariant class ensemble may be written

$$
\rho(\lambda)=\frac{2}{\pi a^{2}} p(\lambda) \sqrt{a^{2}-\lambda^{2}}
$$

with $p(\lambda)$ a polynomial which depends on the ensemble distribution in a complicated way [10]. However, the two-point and higher, connected correlation functions for this class have been found to be universal in form in the limit that $N \rightarrow \infty$, where $N$ denotes the dimension of the matrices [1 4, 6]. Their only dependence on the averaging ensemble is through the endpoint of the eigenvalue spectrum.

In a previous work [3], Brézin and Zee suggested that the two-point and higher correlation functions for the Wigner class (in the $N \rightarrow \infty$ limit) were universal as well, having the same form as those for the invariant class. Here we will show that this suggestion was incorrect, and that the two-point correlation function is somewhat less universal than its invariant class analog, as it depends on two parameters of the distribution (to be defined below) rather than just one. In general the averaged $k$-point connected correlation for a Wigner class ensemble will explicitly depend on the ensemble's first $2 k$ moments, and will be a quantity of order $N^{-3 k / 2+1}$ to $N^{-2 k-2}$ depending on which moments are present.

Amusingly, the occurrence of universality is reversed between these two classes. While the eigenvalue density for the Wigner class is universal, the higher order connected correlation functions increasingly depend on the particulars of the ensemble distribution chosen. In contrast, in the invariant class the eigenvalue density is not universal, but the higher order connected correlation functions are.

For the two-point connected correlation function in the Wigner class (which we calculate for the Hermitian matrix case in the following sections) we find an expression dependent on only the second and fourth moments of the averaging ensemble. Specifically, we obtain 


$$
\rho_{c}(\mu, \nu)=\frac{-1}{2 \pi^{2} N^{2}}\left(\frac{\left(4 \sigma^{2}-\mu \nu\right)}{\beta(\mu-\nu)^{2} \sqrt{\left(4 \sigma^{2}-\mu^{2}\right)\left(4 \sigma^{2}-\nu^{2}\right)}}-\frac{\tau^{4}}{4 \sigma^{8}} \frac{\left(4 \sigma^{2}-2 \mu^{2}\right)\left(4 \sigma^{2}-2 \nu^{2}\right)}{\sqrt{\left(4 \sigma^{2}-\mu^{2}\right)\left(4 \sigma^{2}-\nu^{2}\right)}}\right)
$$

where $\beta=1$, or 2 depending on if one considers real orthogonal or Hermitian matrices, $\sigma^{2}$ is the second moment of the ensemble distribution, $\tau^{4}$ the fourth moment, and $\pm 2 \sigma$ are the endpoints of the eigenvalue spectrum, all to be more explicitly defined later. Within the Wigner class one may consider, for example, such arbitrary and disparate ensemble distributions as $P_{N}\left(\varphi_{i j}\right) \propto \Theta\left(c^{2} / N-\left|\varphi_{i j}\right|^{2}\right), P_{N}\left(\varphi_{i j}\right) \propto \exp \left(-N \sum_{k=1}^{p} g_{k}\left|\varphi_{i j}\right|^{2 k}\right)$, or $P_{N}\left(\varphi_{i j}\right) \propto 1 / 2\left[A \delta\left(\varphi_{i j}-c / \sqrt{N}\right)+B \delta\left(\varphi_{i j}+c / \sqrt{N}\right)\right]$ always obtaining Eq.(1.5).

If we set $\tau^{4}=0$ in Eq.(1.5) we obtain the result for a Gaussian ensemble distribution. This is identical to the universal form of the two-point connected correlation that was obtained by Brézin and Zee for any distribution in the invariant class. The Gaussian case, of course, belongs to both the Wigner and invariant classes. However, in general the two-point correlation for the Wigner and the invariant class differ by an additional term, namely the second term in Eq.(1.5).

In Fig.1 and Fig. 2 we have plotted the functions $\left.(\mu-\nu)^{2} \rho_{c}(\mu, \nu)\right|_{\beta=1}$ and $(\mu-$ $\nu)\left.^{2} \rho_{c}(\mu, \nu)\right|_{\beta=1, \tau^{4} \rightarrow 0}$ with the arbitrary choice $\sigma^{2}=1, \tau^{4}=-2$, and $N=100$ for two specific values of $\mu$. We see that the second term is capable of changing the shape of the curves substantially. Thus, two Wigner systems with identical eigenvalue densities may have very different two-point connected eigenvalue correlations.

In a numerical work Kobayakawa, et. al. [11] found poor agreement between numerical calculations of two-point correlations for specific Wigner ensembles and the universal form for the invariant class. It appears that, with the inclusion of the second term, Eq.(1.5) is in good agreement with the numerical results [1], see Figures 3 and 4 which correspond to (19) and (20) in Ref.].

Recently, we have learned that Pastur, Khorunzhy, and Khoruzhenko [12 have also calculated correlation functions for the general Wigner class, but using a completely different 
technique. We feel it worthwhile to present an independent study, since the techniques we use may be of interest, and the form of the resulting equations we obtain elucidating. Our result (1.5) differs from theirs (Eq.(14) in Ref. [12]) in the sign of the second term.

This article is organized as follows. In Sec. [I we employ a diagrammatic approach to calculate the averaged one, two and three-point Green functions, or resolvents, for general Wigner class ensembles in the large $N$ limit. We then outline how all higher point Green functions may similarly be calculated. In Sec. III we demonstrate a completely different approach to the same problem, namely a renormalization group (RG) inspired procedure for calculating the same Wigner class Green functions. Naturally, the methods of both sections return identical results.

Since from the diagrammatic standpoint this problem takes the form of a large $N$, zero dimensional field theory calculation, it is straightforward to determine the relevant diagrams and sum them. The RG method, on the other hand, takes more work and proves less transparent. However, the RG method makes more apparent the freedom one has in choosing model ensemble densities without changing the resulting correlation functions.

\section{CORRELATIONS VIA DIAGRAMS}

The use of diagrammatic methods in the study of random matrix theories is not new. Such techniques have been successfully applied in both invariant class and Wigner class calculations [3,13] with applications to the latter being limited to a few specific but important ensembles, namely the ones with Gaussian distributed elements.

Here we will show one how may calculate via diagrams the hierarchy of correlation

functions for the Wigner class in general. The known results for Gaussian ensembles will be obtained as a special case. We will begin by explicitly calculating the one and two-point functions before considering the general $n$-point case.

Let $\varphi$ be an element of an ensemble of dimension $N$ matrices with individually identically distributed elements. We limit our consideration to Hermitian matrices, however the method 
may be extended to other sets such as symplectic or real symmetric. Also, we will initially take the case of an even distribution, and save treating the inclusion of odd moments until Sec. IIC.

We choose to define the moments of the matrix element distributions so that the eigenvalues of any matrix will be of order $N^{-1}$ with mean zero and range of order $N^{0}$. If we write $\varphi=X+i Y$, with $X_{j}^{i}=X_{j}^{i}$, and $Y_{j}^{i}=-Y_{i}^{j}$ this is accomplished by defining: $\left\langle X_{j}^{i}\right\rangle=\left\langle Y_{j}^{i}\right\rangle=0$, $\left\langle\left(X_{j}^{i}\right)^{2}\right\rangle=\left(1+\delta_{j}^{i}\right) C_{2} / 2 N,\left\langle\left(Y_{j}^{i}\right)^{2}\right\rangle=\left(1-\delta_{j}^{i}\right) C_{2} / 2 N$, etc. so that $\left\langle\varphi_{j}^{i}\right\rangle=0,\left\langle\varphi_{j}^{i} \varphi_{j}^{i *}\right\rangle=C_{2} / N$, $\left\langle\left(\varphi_{j}^{i} \varphi_{j}^{i *}\right)^{2}\right\rangle-2\left(C_{2} / N\right)^{2}=C_{4} / N^{2}$, etc., where each $C_{n}$ is some constant of order $N^{0}$ and 〈.〉 denotes averaging over the ensemble. Note that in the case of a Gaussian ensemble all moments but $C_{2}$ equal zero.

In general the $k$ th moment is $C_{k} / N^{k / 2}$. The inverse powers of $N$ appear as a result of our requirement that the eigenvalue spectrum be finite in width as $N$ becomes large. Alternatively we might have defined the ensemble density as having moments $C_{2}, C_{4}, \ldots C_{k}$, and the matrices of the ensemble as having elements $\varphi_{j}^{i}=X_{j}^{i} / \sqrt{N}+i Y_{j}^{i} / \sqrt{N}$, with the same effect. We have opted, however, to define the distribution as above in order to facilitate our later counting of the powers of $1 / N$ associated with each diagram.

We define the matrix

$$
\hat{G}_{N}(z)_{j}^{i} \equiv\left(\frac{1}{z-\varphi}\right)_{j}^{i}
$$

and the Green function, or resolvent, as

$$
G_{N}(z) \equiv\left\langle\frac{1}{N} \operatorname{Tr} \hat{G}_{N}(z)\right\rangle
$$

We are interested in $G(z) \equiv \lim _{N \rightarrow \infty} G_{N}(z)$ from which we may extract the averaged eigenvalue density via $\rho(\mu)-\left.\frac{1}{\pi} \operatorname{Im} G(\mu+i \epsilon)\right|_{\epsilon \rightarrow 0}$.

Similarly we define the two-point Green function as

$$
G_{N}(w, z) \equiv\left\langle\frac{1}{N} \operatorname{Tr} \hat{G}_{N}(z) \frac{1}{N} \operatorname{Tr} \hat{G}_{N}(w)\right\rangle
$$

In the large $N$ limit $G_{N}(w, z) \rightarrow G(w) G(z)$; thus we define the connected function as 


$$
G_{c N}(w, z) \equiv G_{N}(w, z)-G_{N}(w) G_{N}(z)
$$

We see that this will be a quantity of order $N^{-2}$. The connected two-point correlation may then obtained via

$$
\rho_{c}(\mu, \nu)=-\frac{1}{4 \pi^{2}}\left[G_{c}(+,+)+G_{c}(-,-)-G_{c}(+,-)-G_{c}(-,+)\right]
$$

where $\left.G_{c}( \pm, \pm) \equiv G_{c}(\mu \pm i \epsilon, \nu \pm i \delta)\right|_{\epsilon, \delta \rightarrow 0}$. Three-point and higher Green functions may be defined, and their corresponding correlation functions obtained in a similar fashion.

\section{A. One point Green function}

To calculate $G(z)$ we consider the untraced average of $\hat{G}(z)$ written as a power series expansion in $1 / z$, and then evaluate the ensemble average of each term in the series. Thus, we start with

$$
G_{N}(z)_{j}^{i}=\sum_{n=0}^{\infty} \frac{1}{z}\left\langle\left[\left(\varphi \frac{1}{z}\right)^{n}\right]_{j}^{i}\right\rangle
$$

The average of any given term in the series may be obtained by summing over all the possible ways of taking the averages of groups of $\varphi$ 's - pairs, triplets, etc. For example, the $n=4$ term of Eq.(2.6) is given by,

$$
\frac{1}{z}\left\langle\left(\varphi \frac{1}{z}\right)^{4}\right\rangle=\frac{1}{z} \hat{\varphi} \frac{1}{z} \hat{\varphi} \frac{1}{z} \tilde{\varphi} \frac{1}{z} \tilde{\varphi} \frac{1}{z}+\frac{1}{z} \hat{\varphi} \frac{1}{z} \tilde{\varphi} \frac{1}{z} \hat{\varphi} \frac{1}{z} \tilde{\varphi} \frac{1}{z}+\frac{1}{z} \hat{\varphi} \frac{1}{z} \tilde{\varphi} \frac{1}{z} \tilde{\varphi} \frac{1}{z} \hat{\varphi} \frac{1}{z}+\frac{1}{z} \hat{\varphi} \frac{1}{z} \hat{\varphi} \frac{1}{z} \hat{\varphi} \frac{1}{z} \hat{\varphi} \frac{1}{z}
$$

where the constituents of a group average are indicated by having either a tilde or hat above them. Given our choice that $\left\langle\varphi_{j}^{i}\right\rangle=0$, single and triple $\varphi$ averages do not contribute to this particular term. It should be emphasized that this procedure entails no approximation

In order to evaluate (2.6) by this procedure we consider it as a diagram expansion and use the double line formalism of 't Hooft [14, where the lines in the diagrams correspond to the indices of the matrices being averaged. Figure 5 shows the diagrams associated with the terms in Eq. (2.7). Each single line corresponds to a $\delta_{j}^{i} / z$ and each double line connects a $\varphi_{j}^{i}$ to one or more others in an average. To borrow from the particle physics literature we 
might call these "quark propagators" and "gluon propagators" respectively. Then two $\varphi$ 's averaged together correspond to an emitted and absorbed gluon. The higher moments of a particular distribution correspond to gluon "interaction vertices" such as the four gluon vertex depicted in Fig. 5(d) [15].

The great advantage of the double line formalism is that it allows one to count the powers of $N$ associated with each diagram with ease. Each continuous closed line represents a sum over the values of an index, and contributes a factor of $N$. Thus, non-planar diagrams, such as Fig. $5(\mathrm{~b})$, are suppressed by powers of $1 / N$ relative to equivalent planar ones since a non-planar diagram always has fewer closed index sums than a planar diagram having the same "interactions".

The nature of the gluon-gluon interaction vertices affords another simplification in the large $N$ limit. Each $k$ th order gluon interaction vertex, such as the fourth order one in Fig. 5(c), contributes a factor of $C_{k} / N^{k / 2}$ (in this way of counting the powers of $1 / N$ associated with the gluon propagators are included). However, any such vertex has only two indices which may be summed over. Figure 6(a) depicts a four-gluon vertex with lines labeled by their index. If such a vertex appears in a diagram, that diagram will have a contribution of order $N^{-(k / 2-1)}$, or less, to the total expansion.

This is the crucial, and simple, reason why the one-point Green function for this class of ensemble is universal. In the large $N$ limit the expansion of Eq. (2.6) only depends on diagrams containing averages of pairs of matrices. In particle physics language we would say that the interactions between the gluons becomes weaker and weaker as $N$ approaches infinity. Thus for large $N$, regardless of the particular features of a distribution, the onepoint Green function and averaged eigenvalue density depend on only one number: the ensemble density's second moment.

This is in direct contrast with the result for invariant class ensembles. Invariant class one-point Green functions are known to depend on the ensemble for which they are calculated in a complicated way [10]. They are completely non-universal. From a diagrammatic standpoint, the key difference between Wigner and invariant ensembles is seen in the gluon 
interaction vertices. The diagram expansion entails summing over all possible ways of taking the the averages of groups of matrices in an expression. In the case of an invariant class problem, enumerating the diagrams to be summed involves perturbatively expanding the ensemble density around the Gaussian case [3]. The perturbations, which are identified with gluon interaction vertices in diagrams, are invariant under the same transformation as the averaging ensemble. Because of this, a $k$ th order gluon interaction vertex will have $k$ indices which are summed on rather than just the two that an analogous Wigner type vertex would have. Such vertices may not be neglected in the large $N$ limit, and by their inclusion the one-point Green function becomes dependent on the distribution in detail. Diagrammatic representations of a simple four-point vertex are displayed in Figure 6 in order to contrast their index structures.

Returning to our Wigner class calculation, the diagram expansion for $G(z)_{j}^{i}$ looks like that in Fig 01(a), in the large $N$ limit. By introducing $\Sigma(z)_{j}^{i}$ as the sum of irreducible diagrams - that is, diagrams which may not be cut on a single quark line to produce two complete and independent ones - we obtain the "integral" equation,

$$
\Sigma(z)_{j}^{i}=\sigma^{2}\left(\frac{1}{z-\Sigma(z)}\right)_{j}^{i}
$$

which is depicted in Fig. G(b). It is obvious from the graphs that $\Sigma(z)_{j}^{i}=\delta_{j}^{i} \Sigma(z)$, thus Eq. (2.8) can be written as a simple quadratic equation. Solving this for $\Sigma$, and using the fact that $\Sigma(z)=\sigma^{2} G(z)$ yields the Green function

$$
G(z)=\frac{1}{2 \sigma^{2}}\left(z-\sqrt{z^{2}-4 \sigma^{2}}\right)
$$

This of course corresponds to the eigenvalue density Eq. (1.3), a long known result. Since it only depends on $\sigma^{2}$ and not on any other details of the ensemble distribution, it holds for any ensemble in the Wigner class, and therefore is known as a universal result. In our further results, however, we will find an increasing dependence on the particulars of the ensemble distribution starting with the two-point connected Green function. 


\section{B. Two-point connected Green function}

We write $G_{c}(w, z)$ as an expansion in $1 / z$ and $1 / w$ with two derivatives taken out explicitly.

$$
\begin{aligned}
N^{2} G_{c}(w, z) & =\frac{1}{w z} \sum_{n=1}^{\infty} \sum_{m=1}^{\infty}\left\langle\operatorname{Tr}\left(\varphi \frac{1}{w}\right)^{n} \operatorname{Tr}\left(\varphi \frac{1}{z}\right)^{m}\right\rangle_{c} \\
& =\frac{\partial}{\partial w} \frac{\partial}{\partial z} \sum_{n=1}^{\infty} \sum_{m=1}^{\infty} \frac{1}{n m}\left\langle\operatorname{Tr}\left(\varphi \frac{1}{w}\right)^{n} \operatorname{Tr}\left(\varphi \frac{1}{z}\right)^{m}\right\rangle_{c},
\end{aligned}
$$

where the subscript $c$ denotes the connected part. In diagram language this expression is a sum of graphs that consist of two different quark loops interacting via one or more gluons. Notice that since we have taken out the two derivatives there are an equal number of $\varphi$ 's and propagators in each trace. The action of each derivative is to sum over all the possible insertions of a quark-quark vertex on it's respective quark loop. The evaluation of the diagrams will be made easier by having taken out these derivatives.

Consider first the case where there are no gluon-gluon interactions, only quark-gluon ones (namely, the Gaussian case), and where contractions are made only between the two traces. In this case then $m$ and $n$ must be equal. If we draw the diagrams so that one loop is inside of another then the planarity of graphs is easy to determine. There are $n$ ways of connecting the first gluon from one loop to another and then only one way of drawing the rest without crossing. Each connection corresponds to averaging together a $\varphi$ in one trace with a $\varphi$ in the other and results in a factor of $\sigma^{2} / N$. In the double line formalism we see each gluon connection creates a new closed index loop and thus contributes a factor of $N$. These graphs sum to

$$
-\frac{\partial}{\partial w} \frac{\partial}{\partial z} \log \left(1-\frac{\sigma^{2}}{w z}\right)
$$

Next consider higher order gluon interactions, still only treating inter-trace connections. Each gluon-gluon interaction vertex of order $k$, corresponding to the averaging together of $k \varphi$ 's split between the traces, contributes a multiplicative factor of $C_{k} / N^{k / 2}$ while adding at most one additional summed index loop. Since $G_{c}(w, z)$ is of order $N^{-2}$, in this case only 
one such graph is allowed, the one with four gluons, two on each loop, interacting via a four point vertex $(k=4)$. This graph, depicted in Fig. 8(a), averages to

$$
\frac{\tau^{4}}{2} \frac{\partial}{\partial w} \frac{\partial}{\partial z}\left(\frac{1}{w z}\right)^{2}
$$

with $\tau^{4} \equiv C_{4}$, the fourth moment of the ensemble distribution.

We must next consider contractions within the same trace. These consist of what could be termed "vertex" and "self energy" corrections. By a vertex correction we refer to the inclusion of gluons which connect two quark lines in the same loop, on either side of a group of one or more quark-gluon vertices. We immediately see that vertex corrections may be ignored since such a gluon turns two closed index loops into one, suppressing the diagram

in which it appears by at least $N^{-2}$. By self energy corrections we refer to the inclusion of gluons emitted and re-absorbed between vertices. The inclusion of self energy corrections to the quarks is instantly achieved through the "dressing" of the bare quark propagators $1 / w$ and $1 / z$ by taking $1 / w \rightarrow G(w)$ and $1 / z \rightarrow G(z)$ everywhere, excepting the partial derivatives.

Thus, we obtain the complete two-point connected Green function for Wigner class ensembles in general,

$$
N^{2} G_{c}(w, z)=-\frac{\partial}{\partial w} \frac{\partial}{\partial z}\left(\log \left[1-\sigma^{2} G(w) G(z)\right]-\frac{\tau^{4}}{2} G(w)^{2} G(z)^{2}\right) .
$$

For the case of Gaussian distributed matrix elements $\tau^{4}=0$ and Eq. (2.11) reduces to the well known result for the Gaussian unitary ensemble (GUE) as we would expect. In general though, we find a dependence on two parameters, the second and fourth moments of the distribution.

\section{Three-point and higher Green functions}

Here we will discuss the application of the diagram method to the calculation of $n$ point connected Green functions. In the previous section we saw that, before the dressing 
procedure, the expansion for the general two-point function differed from that for the GUE by only one diagram. This diagram, once dressed, may be viewed as a correction to the GUE result. As we will see, the higher order connected functions lend themselves to a similar interpretation, and may be written

$$
G_{c N}\left(z_{1}, z_{2}, \ldots, z_{n}\right)=G_{c N}^{(0)}\left(z_{1}, z_{2}, \ldots, z_{n}\right)+(\text { non-Gaussian terms })
$$

where the superscript (0) indicates the GUE result. From here on we will not explicitly calculate the GUE Green functions as they are well known in the literature (see, for example, [13] or [8]). Our main interest will be on the non-Gaussian terms and the diagrams that comprise them.

The relevant, undressed non-Gaussian diagrams are straightforward to enumerate. By our conventions an $n$-point connected Green function for the GUE, as well as the diagrams in its associated expansion, is of order $N^{-2 n+2}$. One creates the non-Gaussian diagrams for a general ensemble by considering gluon interaction vertices; these have a few qualities which greatly reduce the number of relevant diagrams in the large $N$ limit.

First, a $k$ th order gluon interaction vertex $(k>2)$ contributes a multiplicative factor of $C_{k} / N^{k / 2}$ while only having at most two free indices associated with it which may be summed on. Including such a vertex in a connected diagram makes the diagram order $N^{-n-k / 2+2}$ or less. This fact limits the moments that the $n$-point function can depend on to those up to $C_{2 n}$. As we have seen the 2-point function depends only on $C_{2}$ and $C_{4}$, the 3-point function will depend on $C_{2}, C_{4}$ and $C_{6}$, etc. This fact also limits the number of vertices which may appear in any one diagram. In the 2-point case only a single $k=4$ vertex is allowed; the inclusion of another creates a diagram of order $N^{-4}$ or less. In the three-point case we may have a single $k=6$ vertex, or up to two $k=4$ ones.

Second, any two quark loops connected via a gluon interaction vertex may not have any other connections between them to leading order. The simplest case where this may be observed is in the single non-Gaussian diagram of the two-point function, depicted in Fig. 8(a). If we add a single gluon connecting the two loops we obtain Fig. \&(c). This new 
graph is now down by $N^{-1}$ because we have added a gluon contributing $\sigma^{2} / N$ yet created no new sum loops since the gluon interaction vertex requires the lines on either side of it to have the same index value. This effect persists in the general $n$-point connected Green function.

As a demonstration of the use of these simplifying facts we calculate the three-point connected Green function to leading order. From our previous discussion we determine that the non-Gaussian diagrams of the three-point function will depend only on the $C_{4}$ and $C_{6}$ moments and the diagrams in their expansion will have either one 6-point, one four-point, or two four-point gluon interaction vertices [see Fig. 10(a)].

There is only one, undressed diagram containing a 6-point gluon interaction vertex which is relevant to order $N^{-4}$. It corresponds to a term

$$
-\frac{v^{6}}{2 N^{4}} \partial_{1} \partial_{2} \partial_{3}\left(\frac{1}{z_{1} z_{2} z_{3}}\right)^{2}
$$

with $v^{6} \equiv C_{6}$.

In the case where we have a single four-point gluon interaction vertex we have two subcases which are not suppressed: one with the vertex connecting all three loops, and the other with the vertex connection two of the three. With the vertex connecting all three there is only one basic diagram (plus permutations), two gluons connecting to one quark loop and one each to the other two. This corresponds to,

$$
-\frac{\tau^{4}}{2 N^{4}} \partial_{1} \partial_{2} \partial_{3}\left(\frac{1}{z_{1} z_{2} z_{3}}\left(\frac{1}{z_{1}}+\frac{1}{z_{2}}+\frac{1}{z_{3}}\right)\right)
$$

If the four-point vertex only connects two of the quark loops there are an infinite number of graphs. This occurs because, although once we have connected two of the three quark loops via a four-point gluon vertex there may be no other connections between them, either one or the other of the loops may have any number of non-interacting (Gaussian) gluon connections to the third. The resulting diagram expansion will be identical to the expansion of the two-point connected Green function summed over all possible ways to connect a third loop via a four-point gluon vertex. We use the distributive property of the derivative to immediately write this as 


$$
-\frac{\tau^{4}}{N^{4}} \partial_{1} \partial_{2} \partial_{3}\left\{\frac{1}{z_{1}^{2}}\left(\frac{1}{z_{2}} \partial_{2}+\frac{1}{z_{3}} \partial_{3}\right)\left[\log \left(1-\frac{\sigma^{2}}{z_{2} z_{3}}\right)-\frac{\tau^{4}}{2} \frac{1}{z_{2}^{2} z_{3}^{2}}\right]+(1 \leftrightarrow 2)+(1 \leftrightarrow 3)\right\}
$$

A note, relating to the interpretation of this problem as a field theory calculation, is that we would not be able to obtain this sum of diagrams so easily if it were not for the fact we are dealing with a static, zero dimensional field theory. In a field theory with "time" and "space" variables, quark lines would carry momentum which would be conserved at vertices. Then the derivative trick would no longer work.

Finally, one can see that the inclusion of diagrams with two four-point gluon interactions

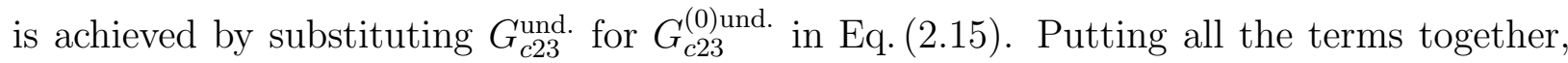
dressing them via $1 / z_{i} \rightarrow G_{i}$, and simplifying some, we find the three-point connected Green function is

$$
\begin{gathered}
N^{4} G_{c 123}=N^{4} G_{c 123}^{(0)}-\partial_{1} \partial_{2} \partial_{3}\left\{\tau^{4} G_{1} G_{2} G_{3}\left(G_{1}+G_{2}+G_{3}\right)+\frac{v^{6}}{2} G_{1}^{2} G_{2}^{2} G_{3}^{2}\right. \\
\left.+\tau^{4}\left[G_{1}^{2}\left(G_{2} \partial_{2}+G_{3} \partial_{3}\right)\left(\log \left(1-\sigma^{2} G_{2} G_{3}\right)-\frac{\tau^{4}}{2} G_{2}^{2} G_{3}^{2}\right)+(1 \leftrightarrow 2)+(1 \leftrightarrow 3)\right]\right\} .
\end{gathered}
$$

\section{D. $n$-point connected Green functions for $C_{n} \neq 0$}

Following the procedure above one may generate the entire hierarchy of connected Green functions. In general,the $n$th connected Green function will equal the GUE $n$-point function plus additional terms depending the ensemble distribution's moments up to $C_{2 n}$ and on powers of the one-point function and its derivatives. However, if the ensemble has an $n$th moment the form of the $n$-point Green function will be far simpler.

Consider our previous example. In the three-point function calculation above we specified an even distribution for the ensemble. If we allow both even and odd moments of the ensemble distribution to exist we must also consider for this case diagrams containing threepoint gluon interaction vertices. We define a third moment of the distribution: $\left\langle\varphi_{j}^{i} \varphi_{i}^{j} \varphi_{j}^{i}\right\rangle=$ $\left(\frac{1+i}{\sqrt{2}}+\left(1-\frac{1+i}{\sqrt{2}}\right) \delta_{j}^{i}\right) C_{3} / N^{3 / 2}$ (no sum on repeated indices). Then, three additional, undressed diagrams, depicted in Fig.10(b), contribute to the three-point connected Green function. 
The second two are order $N^{-4}$ — the same as all the original terms in Eq.(2.16). The first, however, is order $N^{-7 / 2}$. In other words, this single diagram is order $\sqrt{N}$ larger than all the others. Thus, we have

$$
N^{7 / 2} G_{c 123}=-\varsigma^{3} \partial_{1} \partial_{2} \partial_{3}\left(G_{1} G_{2} G_{3}\right)+\mathrm{O}\left(N^{-1 / 2}\right)
$$

where $\varsigma^{3} \equiv C_{3}$.

For an ensemble with a non-zero $n$th moment $(n>2)$ one finds that the $n$-point connected Green function, to leading order, is

$$
N^{3 n / 2-1} G_{c 1 \ldots n}=C_{n} \prod_{i=1}^{n}\left(-\partial_{i}\right) G_{i}+\mathrm{O}\left(N^{-1+1 / 2(n \bmod 2)}\right) .
$$

Thus, depending on the particulars of the ensemble density, the leading order $n$-point connected correlation function can be a quantity of order $N^{-3 n / 2+1}$ through order $N^{-2 n-2}$. This sort of dependence on the existence of various ensemble moments is in striking contrast to the case in Invariant class ensembles. For the Invariant class, 2-point and higher connected correlation functions depend on the ensemble density only through the endpoint of the eigenvalue spectrum and the order in $1 / N$ of the various quantities is, in general, independent of the ensemble density.

\section{RENORMALIZATION GROUP APPROACH}

In this section we demonstrate the use of a renormalization group inspired approach |16] to calculate the connected two-point Green function for theories in the Wigner class. Further application to higher point functions follows in an obvious way.

This method has been used previously, by Brézin and Zee [2], to derive the one-point green function for the Wigner class of theories, as well as by others to derive various quantities in invariant class theories [17]. In the interest of making the somewhat complicated derivation of the two-point function more transparent we will briefly review Brézin and Zee's calculation of the one-point function. 


\section{A. One-point Green function via RG}

The basic idea here is to write the $(N+1)$ dimensional, matrix Green function in terms of dimension $N$ quantities and average over select elements. This generates a partial differential equation which may then be solved in the large $N$ limit.

To begin the derivation of $G(z)$ we write the $(N+1) \times(N+1)$ Hermitian matrix $\varphi_{N+1}$ in the form

$$
\varphi_{N+1}=\left(\begin{array}{cc}
\varphi_{N} & v \\
v^{\dagger} & \chi
\end{array}\right)
$$

with $\varphi_{N}$ an $N \times N$ Hermitian matrix, $v$ a complex $N$ component vector, and $\chi$ a real scalar then the identity

$$
\operatorname{Tr} \frac{1}{z-\varphi_{N+1}}=\operatorname{Tr} \frac{1}{z-\varphi_{N}}+\frac{1+\left\langle v\left|\left(\frac{1}{z-\varphi_{N}}\right)^{2}\right| v\right\rangle}{z-\chi-\left\langle v\left|\frac{1}{z-\varphi_{N}}\right| v\right\rangle}
$$

may be established. One can write this as a partial differential equation for $\hat{G}$,

$$
\operatorname{Tr} \hat{G}_{N+1}(z)=\operatorname{Tr} \hat{G}_{N}(z)+\frac{\partial}{\partial z} \log \left(z-\chi-\left\langle v\left|\hat{G}_{N}(z)\right| v\right\rangle\right)
$$

We obtain a differential equation for $G(z)$ by averaging Eq. (3.2) over the distribution governing $\varphi_{N+1}$, and retaining terms of order $N^{0}$ or larger.

The term on the left hand side of Eq. (3.2) averages to

$$
(N+1) G_{N+1}(z)=(N+1) G_{N}(z)+(N+1) \frac{\partial}{\partial N} G_{N}(z)+\ldots
$$

Since $G_{N}(z)$ is of order $N^{0}$, to the desired order we may neglect all but the first term on the right.

Next, we skip to the second term on the right of Eq. (3.2). Recall that the mean squared value of matrix elements is of order $N^{-1}$. To order $N^{0}$, then, we may immediately set $\chi=0$ in this term. If we expand the logarithm we seen that we will be required to evaluate averages such as $\left\langle v_{i}^{*} v_{j} v_{k}^{*} v_{l} \ldots v_{p}^{*} v_{q}\right\rangle$. Consider the term with four $v$ 's

$$
\left\langle v_{i}^{*} v_{j} v_{k}^{*} v_{l}\right\rangle=A\left(\delta_{i j} \delta_{k l}+\delta_{i l} \delta_{k j}\right)+B \delta_{i j k l}
$$


In the large $N$ limit $A$ terms will dominate, with $A=\left\langle v_{1}^{*} v_{1} v_{2}^{*} v_{2}\right\rangle=\left\langle v_{1}^{*} v_{1}\right\rangle^{2}=\left(\sigma^{2} / N\right)^{2}$ In general every average of a product of $v$ 's will be dominated by second moment terms, and in fact we may replace $\left\langle v\left|1 /\left(z-\varphi_{N}\right)\right| v\right\rangle$ with $\left(\sigma^{2} / N\right) G_{N}(z)$ within the logarithm.

We must be more careful in averaging the first term on the right of Eq. (3.2) since we are averaging over the distribution appropriate for $\varphi_{N+1}$, and not $\varphi_{N}$. Our experience with the second term, which we treated in the previous paragraph, indicates how free our choice of a matrix density may be. First, $G(z)$ depends only on the second moment, $\sigma^{2}$, of the distribution. In fact we may set off diagonal elements to zero with some finite probability without changing $G(z)$. Second, diagonal and off diagonal elements may even obey different distributions since all that is required by this procedure is that $\left\langle\chi^{2}\right\rangle \rightarrow 0$ as $N \rightarrow \infty$.

Thus, if $P\left(\varphi_{i j} ; \sigma^{2} / N, \varsigma^{3} / N^{3 / 2}, \tau^{4} / N^{2}, \ldots\right)$, defines a particular distribution (with $\sigma^{2}, \varsigma^{3}$, $\tau^{4}, \ldots$ being its moments $)$ then it is sufficient to use $P\left(\varphi_{i j} ; \sigma^{2} / N, 0,0, \ldots\right)$ in the calculation of $G(z)$, that is, we may effectively set $\varsigma, \tau, \ldots$ equal to zero. After some straightforward manipulation we see that first term on the right of Eq. (3.2) averages to $N G\left[z ;\left(1-\frac{1}{N}\right) \sigma^{2}\right]+$ $\mathrm{O}\left(N^{-1}\right)$

Putting all this together, we find that in the limit $N \rightarrow \infty$, Eq. (3.2) averages to

$$
G(z)=-\sigma^{2} \frac{\partial}{\partial \sigma^{2}} G(z)+\frac{\partial}{\partial z} \log \left(z-\sigma^{2} G(z)\right)
$$

From dimensional analysis one can see that $G\left(z ; \sigma^{2}\right)=\left(1 / \sigma^{2}\right) G(z / \sigma ; 1)$ and hence $\sigma^{2} \frac{\partial}{\partial \sigma^{2}} G(z)=-\frac{1}{2}\left(1+z \frac{\partial}{\partial z}\right) G(z)$. Finally, we find that Eq. (3.2) becomes

$$
G(z)=z \frac{d}{d z} G(z)+2 \frac{d}{d z} \log \left(z-\sigma^{2} G(z)\right)
$$

This nonlinear equation has the unique solution

$$
G(z)=\frac{1}{2 \sigma^{2}}\left(z-\sqrt{z^{2}-4 \sigma^{2}}\right)
$$

Naturally, this $G(z)$ is identical to that in Eq. (2.9) and thus corresponds to the eigenvalue density (1.3). Now we will apply this method to the problem of deriving the connected two-point Green function. 


\section{B. Two-point connected Green function via RG}

As a starting point, we use Eqs. 2.1) and (3.1) to write

$$
\begin{aligned}
\operatorname{Tr} \hat{G}_{N+1}(w) \operatorname{Tr} \hat{G}_{N+1}(z)= & \operatorname{Tr} \hat{G}_{N}(z) \operatorname{Tr} \hat{G}_{N}(w) \\
& +\frac{\partial}{\partial w} \operatorname{Tr} \hat{G}_{N}(z) \log \left(w-\chi-\left\langle v\left|\hat{G}_{N}(w)\right| v\right\rangle\right) \\
& +\frac{\partial}{\partial z} \operatorname{Tr} \hat{G}_{N}(w) \log \left(z-\chi-\left\langle v\left|\hat{G}_{N}(z)\right| v\right\rangle\right) \\
& +\frac{\partial^{2}}{\partial w \partial z} \log \left(w-\chi-\left\langle v\left|\hat{G}_{N}(w)\right| v\right\rangle\right) \log \left(z-\chi-\left\langle v\left|\hat{G}_{N}(z)\right| v\right\rangle\right) .
\end{aligned}
$$

Now we must average over the distribution governing $\varphi_{N+1}$, keeping only connected terms up to order $N^{-1}$.

The term on the left of Eq. (3.6) is trivially averaged to

$$
(N+1)^{2} G_{c(N+1)}(w, z)=(N+1)^{2} G_{c N}(w, z)+(N+1)^{2} \frac{\partial}{\partial N} G_{c N}(w, z)+\ldots
$$

Since we are keeping only terms up to order $N^{-1}$, and we know that $G_{c N}(w, z)$ itself is order $N^{-2}$, then we may write this as

$$
N^{2} G_{c N}(w, z)+\mathrm{O}\left(N^{-2}\right)
$$

Next we consider the second (and third) term on the r.h.s. of Eq. (3.6). Let us first obtain the connected average to leading order in $1 / N$. To leading order we may immediately set $\chi=0$ and take the average over $v$ inside the logarithm where we see we may again replace $\left\langle v\left|\hat{G}_{N}(w)\right| v\right\rangle$ with $\left(\sigma^{2} / N\right) \operatorname{Tr} \hat{G}(w)$. Thus, we have

$$
-\frac{\partial}{\partial w}\left\langle\operatorname{Tr} \hat{G}_{N}(z) \sum_{n=1}^{\infty} \frac{1}{n}\left(\frac{1}{w} \frac{\sigma^{2}}{N} \operatorname{Tr} \hat{G}_{N}(w)\right)^{n}\right\rangle_{c}
$$

Now we complete the average over the remaining variables, here using the distribution appropriate to $\varphi_{N}$ since corrections are of order $N^{-1}$ and still only concerning ourselves with leading order contributions. To leading order we need only average together $\operatorname{Tr} \hat{G}(z)$ and one of the $\operatorname{Tr} \hat{G}(w)$ 's in each series term. Then $G_{N}(w)$ may be substituted for the remaining $\operatorname{Tr} \hat{G}(w)$ 's. By summing the series, and utilizing the identity 


$$
G_{N}(w)=\frac{1}{w-\sigma^{2} G_{N}(w)}+\mathrm{O}\left(N^{-1}\right)
$$

we obtain

$$
-N \frac{\partial}{\partial w} \sigma^{2} G_{c N}(w, z) G_{N}(w)
$$

as the leading order average of the term in question. Note that this expression is already order $N^{-1}$, therefore we need not consider any corrections to it as they will be order $N^{-2}$ or smaller.

On to the fourth term in Eq. (3.6). To the desired order we retain at most a single second moment in $\chi$, any higher moments or powers of moments may be neglected, yielding

$$
\begin{array}{r}
\frac{\partial^{2}}{\partial w \partial z}\left[\left\langle\sum_{m, n=1}^{\infty} \frac{1}{m n}\left(\frac{1}{w}\left\langle v\left|\hat{G}_{N}(w)\right| v\right\rangle\right)^{n}\left(\frac{1}{z}\left\langle v\left|\hat{G}_{N}(z)\right| v\right\rangle\right)^{m}\right\rangle_{c}\right. \\
\left.+\frac{1}{N} \frac{\sigma^{2}}{z w}\left\langle\left(1+\sum_{n=1}^{\infty} \frac{1}{n} \frac{1}{w^{n}}\left\langle v\left|\hat{G}_{N}(w)\right| v\right\rangle^{n}\right)\left(1+\sum_{m=1}^{\infty} \frac{1}{m} \frac{1}{z^{m}}\left\langle v\left|\hat{G}_{N}(w)\right| v\right\rangle^{m}\right)\right\rangle\right]
\end{array}
$$

In the first term of (3.10) contributions to order $N^{-1}$ will come from one of two connected averages: either

$$
\begin{aligned}
\left\langle v_{i} v_{l}^{*}\right\rangle\left\langle v_{k} v_{j}^{*}\right\rangle \hat{G}(w)_{j}^{i} \hat{G}(z)_{l}^{k} & =\frac{\sigma^{4}}{N^{2}} \operatorname{Tr}[\hat{G}(w) \hat{G}(z)], \text { or } \\
\left\langle v_{i} v_{l}^{*} v_{k} v_{j}^{*}\right\rangle_{c} \hat{G}(w)_{j}^{i} \hat{G}(z)_{l}^{k} & =\frac{\tau^{4}}{N^{2}} \sum_{i=1}^{N} \hat{G}(w)_{i}^{i} \hat{G}(z)_{i}^{i}
\end{aligned}
$$

where $\tau^{4}$ is the fourth moment of the ensemble distribution. Each term is of order $N^{-1}$, thus either one or the other will occur once. The contribution from (3.11) is

$$
\begin{array}{r}
\frac{1}{N} \frac{\partial^{2}}{\partial w \partial z}\left[\frac{1}{w-\sigma^{2} G(w)} \frac{1}{z-\sigma^{2} G(z)}\left\langle\frac{\sigma^{4}}{N} \operatorname{Tr}\left(\hat{G}_{N}(w) \hat{G}_{N}(z)\right)\right\rangle\right] \\
=-\frac{1}{N} \frac{\partial^{2}}{\partial w \partial z}\left[\frac{G(w)-G(z)}{w-z}\right] \sigma^{4} G(z) G(w) \\
=\frac{1}{N} \frac{\partial^{2}}{\partial w \partial z} \frac{\sigma^{4} G^{2}(w) G^{2}(z)}{1-\sigma^{2} G(w) G(z)}
\end{array}
$$

and the contribution from (3.12) is

$$
\frac{1}{N} \frac{\partial^{2}}{\partial w \partial z}\left[\frac{1}{w-\sigma^{2} G(w)} \frac{1}{z-\sigma^{2} G(z)}\left\langle\frac{\tau^{4}}{N} \sum_{i=1}^{N} \hat{G}_{N}(w)_{i}^{i} \hat{G}_{N}(z)_{i}^{i}\right\rangle\right]=\frac{\tau^{4}}{N} G^{2}(w) G^{2}(z)
$$


Treating the second term in (3.10) is far simpler since its average is unconnected and it is manifestly of order $N^{-1}$. We may immediately average it to

$$
\frac{1}{N} \frac{\sigma^{2}}{z w}\left[1+\frac{\frac{\sigma^{2}}{w} G(w)}{1-\frac{\sigma^{2}}{w} G(w)}\right]\left[1+\frac{\frac{\sigma^{2}}{z} G(z)}{1-\frac{\sigma^{2}}{z} G(z)}\right]=\frac{\sigma^{2}}{N} G(w) G(z)
$$

Finally, we come to the first term on the r.h.s. of Eq. (3.6), we have a case where, as in the calculation of $G(z)$, we must carefully average over the distribution appropriate to $\varphi_{N+1}$ and not $\varphi_{N}$. Again we are led to consider how free our choice of probability distribution may be. Consideration of the previous terms has shown that $G_{c N}(w, z)$ will explicitly depend on only two numbers $\sigma^{2}$ and $\tau^{4}$, thus it is sufficient to average this term using the general distribution $P\left(\varphi_{i j} ; \sigma^{2} / N, \tau^{4} / N^{2}\right)$.

We find the term in question then averages to

$$
\left[1-\frac{1}{N}\left(\sigma^{2} \frac{\partial}{\partial \sigma^{2}}+2 \tau^{4} \frac{\partial}{\partial \tau^{4}}\right)\right] G_{c N}(w, z)
$$

From dimensional analysis one can see that

$$
G_{c N}\left(w, z ; \sigma^{2}, \tau^{4}\right)=\frac{1}{\sigma^{2}} G_{c N}\left(w / \sigma, z / \sigma ; 1, \tau^{4} /\left(\sigma^{2}\right)^{2}\right)
$$

Hence, the identity

$$
\sigma^{2} \frac{\partial}{\partial \sigma^{2}} G_{c N}(w, z)=-\left(1+\frac{z}{2} \frac{\partial}{\partial z}+\frac{w}{2} \frac{\partial}{\partial w}+2 \tau^{4} \frac{\partial}{\partial \tau^{4}}\right) G_{c N}(w, z) .
$$

Using this we see that the first term on the r.h.s. of Eq. (3.6) finally averages to

$$
\left(1+\frac{1}{2} \frac{\partial}{\partial w} w+\frac{1}{2} \frac{\partial}{\partial z} z\right) N^{2} G_{c N}(w, z)+\mathrm{O}\left(N^{-2}\right)
$$

Using expressions (3.7), (3.9), (3.14), (3.15) and (3.16) we obtain the partial differential equation

$$
\left[\sum_{i=1,2} \partial_{i}\left(z_{i}-2 \sigma^{2} G_{i}\right)\right] N^{2} G_{c 12}=-2 \partial_{1} \partial_{2}\left(\frac{\sigma^{2} G_{1} G_{2}}{1-\sigma^{2} G_{1} G_{2}}+\tau^{4} G_{1}^{2} G_{2}^{2}\right)+\mathrm{O}(1 / N),
$$

where we have used the obvious shorthand: $G_{c 12} \equiv G_{c}(w, z), \partial_{1} \equiv(\partial / \partial w), G_{1} \equiv G(w)$, etc. Note that the derivatives on the l.h.s. act on $G_{c 12}$ as well as on the parenthesized terms immediately to their right. 
This rather complicated, non-linear equation is solvable. In general we may write its solution in the form

$$
N^{2} G_{c 12}=\partial_{1} \partial_{2} F\left(G_{1}+G_{2}, G_{1}-G_{2}\right)+h_{1}\left(G_{1}\right)+h_{2}\left(G_{2}\right)
$$

with $F, h_{1}$, and $h_{2}$ unknown functions. We may immediately set $h_{1,2}=0$ since $G_{c 12}$ must go like $1 / z_{1,2}^{2}$ as $z_{1,2} \rightarrow \infty$. We substitute this general solution into Eq. (3.17) and use the large $N$ identity, $G / G^{\prime}=2 \sigma^{2} G-z$, where $G^{\prime} \equiv(\partial / \partial z) G(z)$,

$$
\partial_{1} \partial_{2}\left[\left(\frac{G_{1}}{G_{1}^{\prime}} \partial_{1}+\frac{G_{2}}{G_{2}^{\prime}} \partial_{2}\right) F\left(G_{1}+G_{2}, G_{1}-G_{2}\right)\right]=\partial_{1} \partial_{2}\left[\frac{2 \sigma^{2} G_{1} G_{2}}{1-2 \sigma^{2} G_{1} G_{2}}+\tau^{4} G_{1}^{2} G_{2}^{2}\right] .
$$

The structure of the equation suggest that we make the variable substitution $g_{ \pm} \equiv$ $\frac{1}{\sqrt{2}}\left(\log G_{1} \pm \log G_{2}\right)$. We equate the bracketed terms in Eq. (3.19) to obtain

$$
\sqrt{2} \frac{\partial}{\partial g_{+}} F\left(e^{g_{+}}, e^{g_{-}}\right)=\frac{2 \sigma^{2} e^{g_{+}}}{1-\sigma^{2} e^{g_{+}}}+2 \tau^{4} e^{2 g_{+}}
$$

which is trivially integrable. Thus,

$$
N^{2} G_{c 12}=-\partial_{1} \partial_{2}\left[\log \left(1-\sigma^{2} G_{1} G_{2}\right)-\frac{\tau^{4}}{2} G_{1}^{2} G_{2}^{2}\right]+f\left(G_{1} / G_{2}\right)
$$

where $f$ is an unknown function. The known asymptotic behavior of $G_{c 12}$ requires $f=0$, thus the unique solution to Eq. (3.17) is

$$
N^{2} G_{c}(w, z)=-\frac{\partial}{\partial w} \frac{\partial}{\partial z}\left(\log \left[1-\sigma^{2} G(w) G(z)\right]-\frac{\tau^{4}}{2} G(w)^{2} G(z)^{2}\right)
$$

By Eq.(2.5) we find the corresponding correlation function is $\rho_{c}(\mu, \nu)=\frac{-1}{4 \pi^{2} N^{2}} \frac{\partial}{\partial \mu} \frac{\partial}{\partial \nu}\left(\log \frac{4 \sigma^{2}-\mu \nu+\sqrt{\left(4 \sigma^{2}-\mu^{2}\right)\left(4 \sigma^{2}-\nu^{2}\right)}}{4 \sigma^{2}-\mu \nu-\sqrt{\left(4 \sigma^{2}-\mu^{2}\right)\left(4 \sigma^{2}-\nu^{2}\right)}}-\frac{\tau^{4} \mu \nu}{2 \sigma^{8}} \sqrt{4 \sigma^{2}-\mu^{2}} \sqrt{4 \sigma^{2}-\nu^{2}}\right)$,

which corresponds, when differentiated, to the previously displayed Eq. (1.5). We simplify by a change of variables to $\sin \theta \equiv \mu / 2 \sigma, \sin \phi \equiv \nu / 2 \sigma$ which allows us to write

$$
\rho_{c}(\theta, \phi)=\frac{-1}{16 \pi^{2} \sigma^{2} N^{2} \cos \theta \cos \phi} \frac{\partial}{\partial \theta} \frac{\partial}{\partial \phi}\left(\log \frac{1+\cos (\theta+\phi)}{1-\cos (\theta-\phi)}-\frac{2 \tau^{4}}{\sigma^{4}} \sin 2 \theta \sin 2 \phi\right)
$$


We can compare this to the known result for invariant class Hermitian ensembles

$$
\rho_{c}(\theta, \phi)=\frac{-1}{4 \pi^{2} a^{2} N^{2} \cos \theta \cos \phi} \frac{\partial}{\partial \theta} \frac{\partial}{\partial \phi} \log \frac{1+\cos (\theta+\phi)}{1-\cos (\theta-\phi)}
$$

where $a$ is the endpoint of the eigenvalue spectrum and depends in detail on the ensemble distribution. The Wigner class result is slightly "less universal" in that it has an additional term and depends on a second number, $\tau^{4}$. As we have seen, higher order connected Wigner correlation functions will depend on more and more moments of their ensemble distribution.

\section{CONCLUSION}

For Wigner class ensembles, we have derived a general form for the one, and two-point connected correlation functions using both a diagrammatic and a renormalization group approach. Using the diagrammatic approach, we have also shown how one may construct higher order correlation functions, in the large $N$ limit. By the RG inspired method we have demonstrated that results for the one and two-point functions are unchanged by considering some general cases where the elements of matrices in the ensemble are not identically distributed. For instance, one may set elements to zero with some finite probability or allow on and off diagonal elements to have different distributions without changing the final result.

In general the $n$-point function will depend on the ensemble's moments up to $C_{2 n}$ and be comprised of products and derivatives of the universal one-point Green function. We have found, though, in the case where a particular ensemble distribution has a non-zero $n$th moment the $n$-point connected Green function is particularly simple to leading order. This contrasts with the case for invariant class ensembles, where the one-point Green function depends on the ensemble in detail while higher order connected Green functions are universal in form. 


\section{ACKNOWLEDGMENTS}

We thank Roland Speicher for calling our attention to the work of Khorunzhy, et.al. in Ref. [12]. One of us (AZ) thanks E. Brézin for discussions on this and other related issues in random matrix theory. We are grateful to Joshua Feinberg for reading this manuscript. This work was supported in part by the National Science Foundation under Grant No. PHY 89-04035. 


\section{REFERENCES}

[1] E. Brézin and A. Zee, Nuc. Phys. B 402(FS), 613 (1993).

[2] E. Brézin and A. Zee, Compt. Rend. Acad. Sci. 17735 (1993).

[3] E. Brézin and A. Zee, Phys. Rev. E 49, 2588 (1994).

[4] E. Brézin and A. Zee, Nuc. Phys. B 424, 435 (1994).

[5] J. D’Anna, E. Brézin and A. Zee,Nuc. Phys. B 443 (1995) 433-443.

[6] C.W.J. Beenakker, Nuc. Phys. B 422, 515, 1994; for earlier work, see C.W.J. Beenakker, Phys. Rev. Let. 70, 1155 (1993).

[7] P.J. Forrester, Nuc. Phys. B 435, 421 (1995).

[8] B. Eynard, SPhT/93-45.

[9] E. Wigner, Can. Math. Congr. Proc. p.174 (University of Toronto Press) and other papers reprinted in C.E. Porter, Statistical Theories of Spectra: Fluctuations (Academic Press, New York, 1965).

[10] E. Brézin, C. Itzykson, G. Parisi, and J. B. Zuber, Commun. math. Phys. 59, 35 (1978).

[11] T.S. Kobayakawa, Y. Hatsugai, M. Kohmoto, and A. Zee, Phys. Rev. E 51, 5365 (1995).

[12] A. Khorunzhy, B. Khoruzhenko, and L. Pastur,J. Phys. A 28, L31-L35(1995).

[13] J. Verbaarschot, A Weidenmüller, and M. Zirbauer, Ann. of Phys. 153367 (1984).

[14] G. 't Hooft, Nuc. Phys. B 72, 461, 1974.

[15] We choose this language for its convenience and familiarity but the reader should keep in mind that there is no $U(N)$ symmetry here so our use of the term "gluon" is somewhat casual. Also, what we, for simplicity's sake, call a $k$-point gluon interaction vertex might more precisely be called an un-amputated, $k$-point gluon Green function. 
[16] E. Brézin and J. Zinn-Justin, Phys. Lett. B 288,54 (1992).

[17] S. Higuchi, C. Itoi, S. Nishigaki, and N. Sakai, Nuc. Phys. B 434, 283 (1995). 


\section{FIGURES}

FIG. 1. The function $\left.(\mu-\nu)^{2} \rho_{c}(\mu, \nu)\right|_{\mu=0}$ for the Wigner class with parameters $\beta=1, \sigma^{2}=1$, and $N=100$, plotted in the two cases $\tau^{4}=0$ (solid line) and $\tau^{4}=-2$ (dashed line).

FIG. 2. The function $\left.(\mu-\nu)^{2} \rho_{c}(\mu, \nu)\right|_{\mu=1}$ for the Wigner class with parameters $\beta=1, \sigma^{2}=1$, and $N=100$, plotted in the two cases $\tau^{4}=0$ (solid line) and $\tau^{4}=-2$ (dashed line).

FIG. 3. Fig. (19) from Ref. [11]. Dashed line is a numerically obtained smoothed connected correlation $\left.(\mu-\nu)^{2} \rho_{c}(\mu, \nu)\right|_{\mu=0}$ for a real symmetric Wigner class ensemble with matrix elements chosen to be $\pm 1 / \sqrt{N}$ with equal probability, and $N=100$. Solid line is $(\mu-\nu)^{2}$ times the universal connected correlation for real symmetric invariant class ensembles with $N=100$, and spectral endpoints \pm 2 , at $\mu=0$.

FIG. 4. Fig. (20) from Ref. [11]. Dashed line is a numerically obtained smoothed connected correlation $\left.(\mu-\nu)^{2} \rho_{c}(\mu, \nu)\right|_{\mu=1}$ for a real symmetric Wigner class ensemble with matrix elements chosen to be $\pm 1 / \sqrt{N}$ with equal probability, and $N=100$. Solid line is $(\mu-\nu)^{2}$ times the universal connected correlation for real symmetric invariant class ensemble, in the case $N=100$, and spectral endpoints \pm 2 , at $\mu=1$.

FIG. 5. The double line diagrams for terms in the expansion of $G(z)$ which have four $\varphi$ 's in them.

FIG. 6. Wigner class versus invariant class vertices.

FIG. 7. Diagram expansions in the limit of large $N$ for: (a) the one-point Green function, and (b) the sum of one-point irreducible graphs.

FIG. 8. Various connected quark loops with interacting gluons. Graph (a) is of order $N^{0}$, while (b) and (c) are of order $N^{-1}$, and (d) is $N^{-2}$. 
FIG. 9. Graphical representation of the expansion of the "undressed" two-point connected Green function

FIG. 10. (a) Diagrams representing the undressed Gaussian 3-point connected Green function for an even ensemble distribution, and (b) additional diagrams arising when odd moments are allowed. 